\title{
Factors Affecting the Segmentation of the Heart Ventricles in Short Axis Cardiac Perfusion MRI Images
}

\author{
Doaa Mousa ${ }^{1}$, Nourhan Zayed, Inas A. Yassine \\ Computers and Systems Department, Electronics Research Institute, Cairo, Egypt \\ Doaa2590@eri.sci.eg \\ Computers and Systems Department, Electronics Research Institute, Cairo, Egypt \\ nourhan@eri.sci.eg \\ Systems and Biomedical Engineering Department, Faculty of engineering, Cairo University, \\ Giza, Egypt. \\ inas.yassine@gmail.com
}

\begin{abstract}
Cardiovascular diseases (CVDs) cause $31 \%$ of the death rate globally. Automatic accurate segmentation is needed for CVDs early detection. In this paper, we study the effect of the registration and initialization of the level set segmentation on the performance of extracting the heart ventricles for the short axis cardiac perfusion MRI images, as a result, we propose a modified workflow to automatically segment the ventricles by mitigating the levelset initial contour extraction in order to improve the segmentation results accuracy. In the registration experiments, the translational transformation was studied based on both the spatial and frequency domain. The frequency domain based registration is mainly established based on the phase correlation methodology. As for the segmentation experiments, the level set initialization was done through extracting the ventricles' real shape from each slice. Though, the final contour of any frame will be used as the initial contour for the next frame. The second initialization strategy was based on defining the initial contour for each frame using the polar representation of the image. Two short axis view datasets of cardiac magnetic resonance (CMR) perfusion imaging were used in testing the proposed methods. Dice coefficient, sensitivity, specificity and Hausdorff distance have been used to evaluate and validate the segmentation results. The system workflow consists of five main modules: preprocessing, localization, initial contour extraction, registration, and segmentation. The segmentation accuracy for left and right ventricles improved from $72 \%$ to $77 \%$ and from $70 \%$ to $81 \%$ using the spatial domain based registration algorithm. The polar-based initialization strategy improves the segmentation accuracy from $77 \%$ to $81 \%$ and from $81 \%$ to $82 \%$ for the left and right ventricles respectively.
\end{abstract}

\section{Indexing terms/Keywords}

Cardiovascular diseases (CVDs), Segmentation, Active contour, Registration, Level set initialization, Perfusion MRI.

\section{Academic Discipline And Sub-Disciplines}

Medical Imaging; Biomedical Image processing.

\section{INTRODUCTION}

Cardiovascular diseases (CVDs) are the class of diseases related to heart and blood vessels such as: Coronary heart disease, Stroke, Cardiomyopathy, etc. CVDs cause $31 \%$ of the death rate globally [1]. Though, an international increase in the focus and awareness for the need of early diagnosis procedures. Cardiac Magnetic Resonance Imaging (CMR) has been widely adopted to diagnose CVD patients' in clinical routine. It provides high-resolution imaging and clear tissue contrast in addition to avoiding the exposure to the ionizing radiation. Perfusion CMR includes the injection of a special dye, often called contrast agent, during the scan in order to highlight the blood pool when good blood supply reaches it, which increases the contrast between muscle and blood pool [2]

Accurate segmentation is the next important step in the workflow of cardiac function evaluation process. Ejection fraction $(E F)$, left ventricle volume, and muscle wall thickness are some of the parameters need to be measured precisely to evaluate the cardiac function. Calculation of these parameters requires an accurate delineation of muscles and ventricles contours. Manual heart contouring by expertise is one solution to this problem but it is an extremely tedious task, time consuming, and highly affected by the observer variability. Therefore fully automatic segmentation is an important Moreover, this task is considered challenging due to the high datasets variability caused by MR scanners, motion artifacts, different noise sources, poor contrast between left ventricle (LV) and myocardium and complex shape of right ventricle $(\mathrm{RV})$. In this study, we will focus our study on the effect of the registration and the active contour initialization on the accuracy of the ventricles segmentation task. The Left Ventricle (LV) segmentation meets lots of challenges due to the huge variation in size and shape of the LV due to the slice position as well as the heart size variability, the motion artifacts as well as the effect of papillary muscles. The RV segmentation is considered more challenging than the LV as it suffers from the complex crescent structure, wall irregularities, inhomogeneity and its ill-defined borders [3].

Several techniques are proposed to perform cardiac segmentation using active contour. Pluempitiwiriyawej et al. [4] introduced an automatic initialization approach for the myocardium segmentation, based on thresholding and morphological operations. Weijia et al. [5] proposed a scheme for automatic close to boundary initialization for deformable models. They generate the initial contour through a labeling/recognition process using Topographic Independent Component Analysis (TICA) learning and feature exaction technique. Wu et al. [6] improved the LV segmentation accuracy by adding a circle-shape based term to the active contour energy function, representing the LV shape. Li et .al [7] proposed a distance regularization term for the level set algorithm in order to maintain the regularity of the level set function during its evolution. Khalifa et al. [8] proposed a framework based on registration and level set method to segment
$7218 \mid$ P a g e
council for Innovative Research
August 2016
w w w. cirworld.com 
the LV, based on the affine-based registration followed by a local B- splines based alignment, to account for local and global motion of the heart. Mousa et al. discussed the impact of polar-based initialization and frame time curve selection in the LV segmentation [9].

Motivated by the challenges in the initialization and registration on the segmentation accuracy, we propose studying the effect of these tasks on the ventricles segmentation tasks. This study is achieved through developing a modified workflow to automatically segment the LV and RV using level set method. The use of level set theory has provided more flexibility and convenience in the implementation of active contours. To the best of our knowledge, the RV segmentation was not studied for perfusion MRI due its complexity.

\section{MATERIALS AND METHODS}

\subsection{Datasets}

Two short axis cardiac magnetic resonance (CMR) perfusion datasets have been used for the segmentation performance evaluation in this study. The datasets were acquired using the Gradient Recalled-Echo (GR) MR scan protocol. It consists of 10 image sequences for 6 different patients. Each sequence is formed of 3 slices named; Basal, medial, and epical for different time frames ranged between 32 and 101 time frame.

\subsection{Experimental Work}

Our study consists of five main steps: Preprocessing, Heart Localization, Initial Contour Extraction, Registration, and Segmentation.

\subsubsection{Preprocessing and localization step}

The aim of the denoising step is to enhance the image quality without affecting image details. Firstly, the MR images are filtered using Gaussian filter in order to remove the noise while preserving the image's edges, as the MRI imaging related noise could be assumed to follow the Gaussian distribution [10]. The images are then analyzed to define the heart region, considered as the region of interest (ROI), through assuming the heart ventricles as two intersected circles (localization step). In our study, we used the Circular Hough transform to search for the LV as well as the RV [11].

\subsubsection{Initial contour extraction}

To the best of our knowledge, most of the segmentation algorithms in the literature use a circular or elliptic shape in the center of the slice as the initial contour for the LV $[3,4,6]$. Nevertheless, these assumptions will fail specially in case of abnormality and muscle deficiency. In addition, it is considered an inappropriate assumption for RV shape. Therefore after Finding the ROI (localization step), we propose finding an initial contour that roughly reflects the shape, size and position of the LV and RV heart related to each slice. In this step, we propose using the intensity time curve information for initialization. The proposed algorithm (Algorithm 1) can be summarized as follows:

a) The intensity time curve, shown in Figure 1, is calculated for the ROI selected by the localization step for each slice. The intensity time curve is defined as the average high intensities in each time frame, the high intensity is defined as the intensity greater than the threshold $(T)$ calculated as the maximum of threshold calculated based on Otsu' method and intensity value calculated at $80 \%$ of the area under intensity probability density function curve.

b) The best frame is selected from the intensity time curve through sorting the peaks of the first one third of the cardiac cycle. The peaks of the intensity time curve reflect the frames with high intensities due to the maximum filling of the contrast agent in RV and LV respectively, occurring at the first one third of the cardiac cycle. Therefore, the best frames to extract the initial contour for both RV and LV respectively are founded in this period.

c) The selected frame is converted from gray level to binary image using the threshold $T$.

d) Finally, a series of morphological operations (opening, closing) were used to remove unwanted pixels. The initial extracted contour is the contour of remaining object, as shown in Figure 2.

Algorithm 1: Initial Contour Extraction
1: input: ROI time frames related to each slice
2: output: initial contour for LV, and RV
3: calculate the intensity time curve for the whole slice
4: detect the peaks of the curve
5: save peaks at first one third of the curve time
6: select the max. two peaks (first one, in location, related to best frame for RV, and the
other to LV frame)
7: threshold the selected frame using T value
8: remove unwanted pixels using morphological operations
9: the largest connected object contour is target contour

$\begin{array}{rrr}7219 & \text { Page } & \text { council for Innovative Research } \\ \text { August } 2016 & \text { www.cirworld.com }\end{array}$




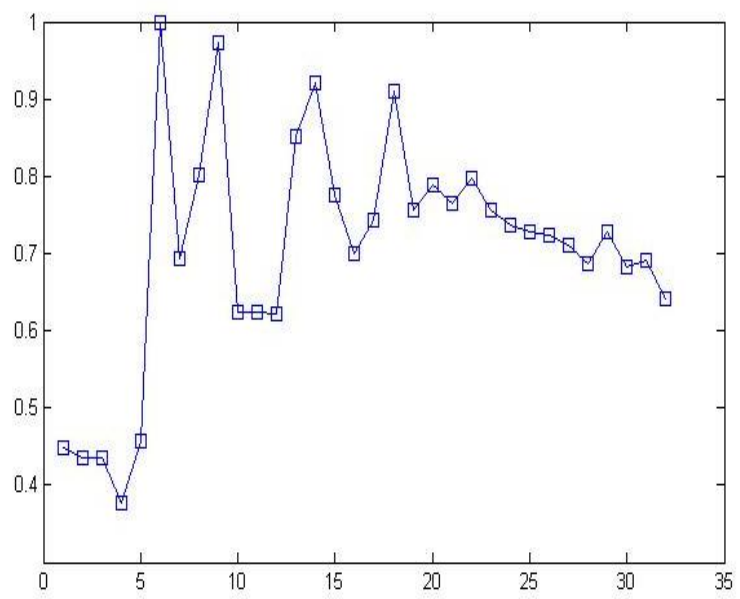

Fig 1: the intensity time curve

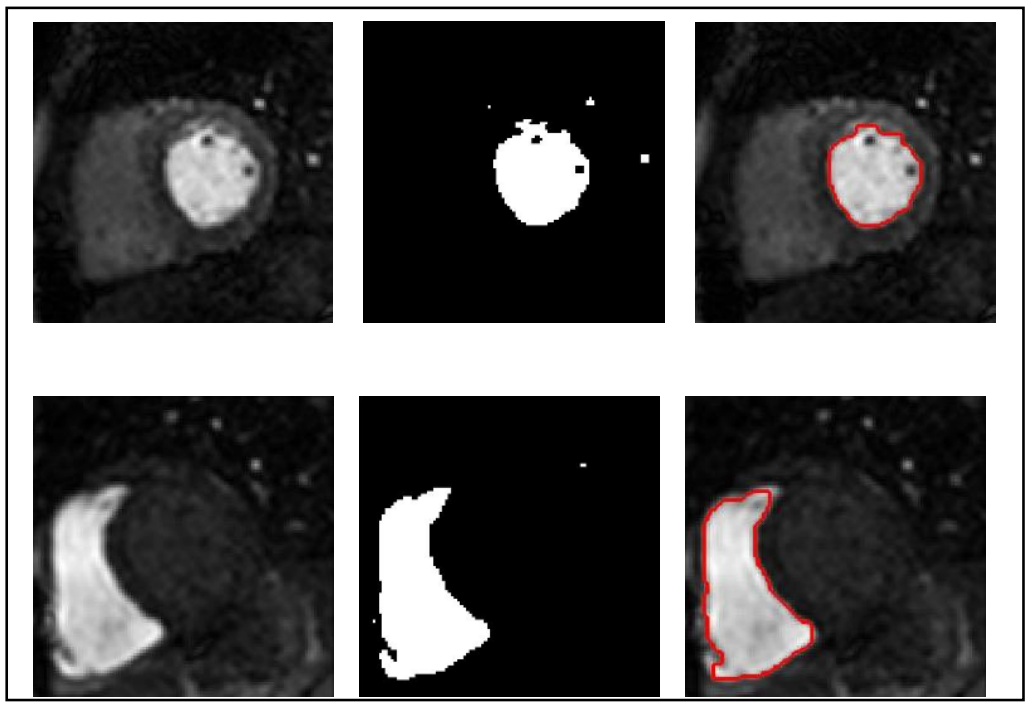

Fig 2: Initial contour extraction steps for LV (first row), and RV (second row)

\subsubsection{Registration step}

It is the process of aligning all time frames to the automatically selected reference frame for each slice. Though, two different registration algorithms were studied, to highlight the effect of the registration on the accuracy of segmentation. For simplicity, the translational transformation is the only studied, based on both spatial and frequency domains. The frequency based registration is mainly calculating the Fourier shift between 2 images corresponding to that in the spatial domain, which is represented formed as a linear phase difference. Though, the phase correlation between two images $I_{1}$ and $\mathrm{I}_{2}$ can be defined as the normalized cross power spectrum between $A$ and $B$ as in the following equation:

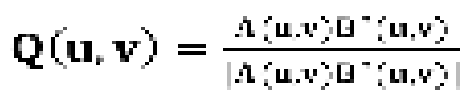

where A, B are the Fourier transform of $\mathrm{I} 1$ and $\mathrm{I} 2$ respectively [12].

\subsubsection{Segmentation using active contour}

The aim of segmentation is to extract the LV and RV using the level set algorithm [13]. This algorithm requires a well localized initial contour in order to accurately and rapidly perform the segmentation task.

\subsubsection{Contour initialization}

In our study, we will investigate 2 different methodologies for the contour initialization process to study their effect of on the segmentation accuracy. The first initialization is considering the previously extracted contour from each slice is considered as the starting initial contour for this slice. In addition, the final contour segmented for any time frame is considered as the initial contour for the next frame. In the second initialization algorithm, we propose defining initial contour for each frame 
based on the image's polar representation. The algorithm can be defined as follows:

a) Each Cartesian frame is transformed into its polar coordinates representation where the center of transformation is calculated from the previously extracted initial contour.

b) The gray level polar coordinates image is converted to a binary image using a threshold T value for the LV and T1 value for the RV; where T1 is the average value of threshold using Otsu' method and intensity value at $80 \%$ of the area under probability density function curve.

c) The objects, found in the upper first third of the binary image, are kept; as the transformation process locate the LV region at top third of the image (approximately the height of LV to image height). This step used only for LV.

d) The image is then transformed back from the polar to the Cartesian coordinate, where the contour of survived object is considered as the initial contour of this frame as shown in Figure 3, and Figure 4.

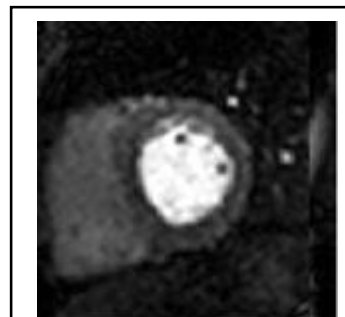

Image in Cartesian

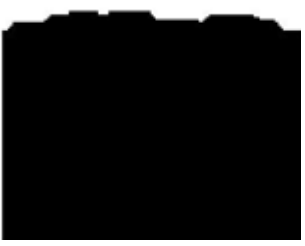

After processing

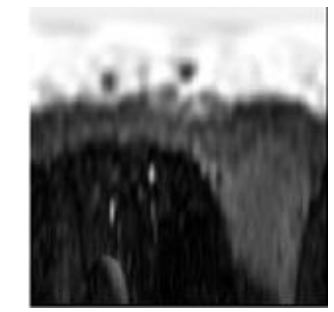

Transformed image to polar

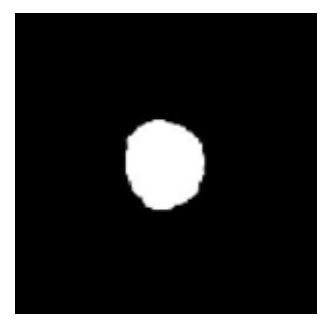

Image back to Cartesian Initial contour superimposed on original image

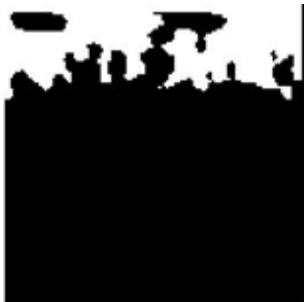

After threshold

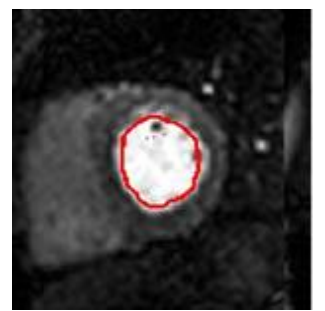

Fig 3: Initial contour extraction for LV

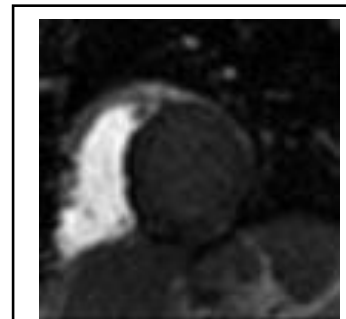

Image in Cartesian

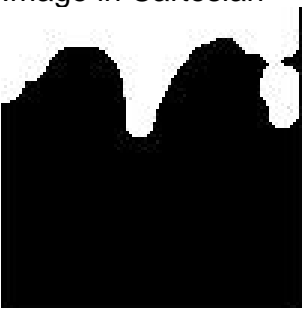

After processing

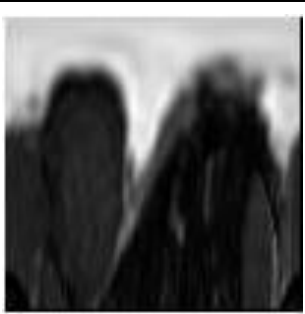

Transformed image to polar

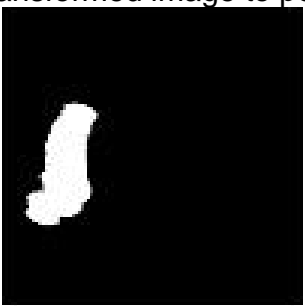

Image back to Cartesian

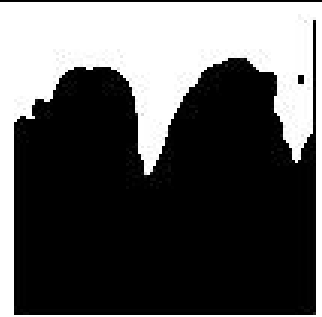

After threshold

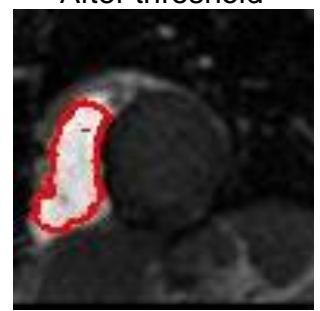

Initial contour superimposed on original image

Fig 4: Initial contour extraction for LV

\subsubsection{Level set method}

After obtaining the initial contour, the Distance Regularized Level Set Evolution (DRLSE) is used for the segmentation task. It allows the use of relatively large time steps to significantly speed up curve evolution, while ensuring adequate numerical accuracy [7]. 


\subsubsection{Evaluation metrics}

In order to evaluate the performance of segmentation, Dice metric, and Hausdorff distance were used to compare the segmented contour to experts segmented contour [14].

\section{RESULTS}

The performance of the proposed method was tested using two datasets of CMR perfusion images. The different registration algorithms effect on the segmentation of LV and RV is shown in Table 1 \& Figure 5, and Table 2 \& Figure 6 respectively. The use of straightforward translation concept in image domain gives better result than that in the frequency domain using phase correlation method. The phase correlation is highly affected by image noise as it produces high peaks in Fourier transform images. In order to improve the results Gaussian filter was used to smooth the Fourier image. This process still failed to succeed in registering some frames. Though, it degrades the overall segmentation accuracy. The difference in accuracy due to effect of registration is about 5\%, and $9 \%$ for LV and RV respectively.

Table 3, Figure 7 and Table 4, Figure 8 show the effect of the initialization to level set method using the previously extracted contour and polar based initialization algorithms on the performance of the segmentation of the LV and RV respectively. From results, we can conclude that the initialization process affects the segmentation accuracy. Changing the initialization method affect the accuracy of segmentation by factor of $4 \%$ and $1 \%$ when applied for the segmentation of LV and RV respectively. The polar-based method improves the result through extracting initial contour for each frame instead of taking it from the previous frame as done in the literature.

The average accuracy of the proposed segmentation algorithm has been improved from 0.77 to 0.82 and from 6.8 to 6.3 , measured using Dice metric (DM) and Hausdorff (HD) similarity metrics respectively, as seen in Figure 9 shows examples of our segmentation results.

Table 1. Effect of registration on LV segmentation using levelset method. The results obtained using first way of initialization as mentioned before.

\begin{tabular}{|l|c|c|c|c|c|}
\hline & Slice level & $\begin{array}{c}\text { DM (mean } \\
\pm \text { SD) }\end{array}$ & $\begin{array}{c}\text { HD } \\
\text { (mean } \pm \text { SD) }\end{array}$ & Sensitivity (\%) & Specificity (\%) \\
\hline $\begin{array}{l}\text { Translation in } \\
\text { image domain } \\
\text { (Reg.1) }\end{array}$ & Base & $0.89 \pm 0.08$ & $5.77 \pm 3.24$ & 84.04 & 99.34 \\
\cline { 2 - 6 } & Mid & $0.73 \pm 0.08$ & $9.10 \pm 2.49$ & 66.20 & 99.34 \\
\hline $\begin{array}{l}\text { Translation in } \\
\text { frequency } \\
\text { domain } \\
\text { (Reg.2) }\end{array}$ & Base & $0.70 \pm 0.13$ & $5.69 \pm 2.25$ & 79.38 & 99.77 \\
\cline { 2 - 6 } & Mid & $0.75 \pm 0.10$ & $8.54 \pm 3.49$ & 85.75 & 97.92 \\
\hline
\end{tabular}

Table 2. Effect of registration on RV segmentation using levelset method. The results obtained using first way of initialization as mentioned before.

\begin{tabular}{|l|c|c|c|c|c|}
\hline & Slice level & $\begin{array}{c}\text { DM } \\
\text { (mean } \pm \text { SD) }\end{array}$ & $\begin{array}{c}\text { HD } \\
\text { (mean } \pm \text { SD) }\end{array}$ & Sensitivity (\%) & Specificity (\%) \\
\hline $\begin{array}{l}\text { Translation in } \\
\text { image domain }\end{array}$ & Base & $0.86 \pm 0.06$ & $10.24 \pm 3.06$ & 91.86 & 96.79 \\
\cline { 2 - 6 } & Mid & $0.77 \pm 0.09$ & $10.03 \pm 2.86$ & 71.59 & 98.97 \\
\hline $\begin{array}{l}\text { Translation in } \\
\text { frequency } \\
\text { domain }\end{array}$ & Base & $0.80 \pm 0.08$ & $11.29 \pm 4.51$ & 89.21 & 47.06 \\
\cline { 2 - 6 } & Mid & $0.59 \pm 0.12$ & $13.70 \pm 3.84$ & 95.99 & 97.35 \\
\hline
\end{tabular}




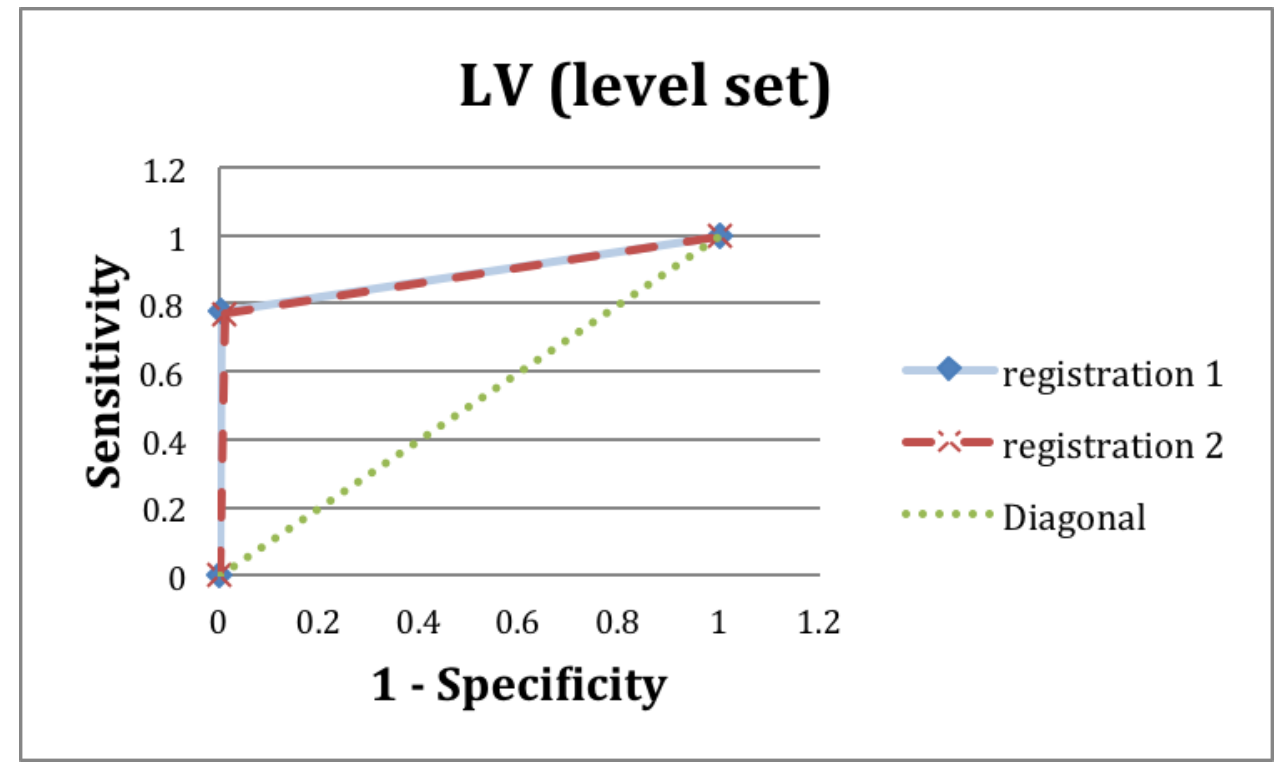

Fig 5: the ROC curve defines the difference between the two registration algorithms on LV segmentation using level set method

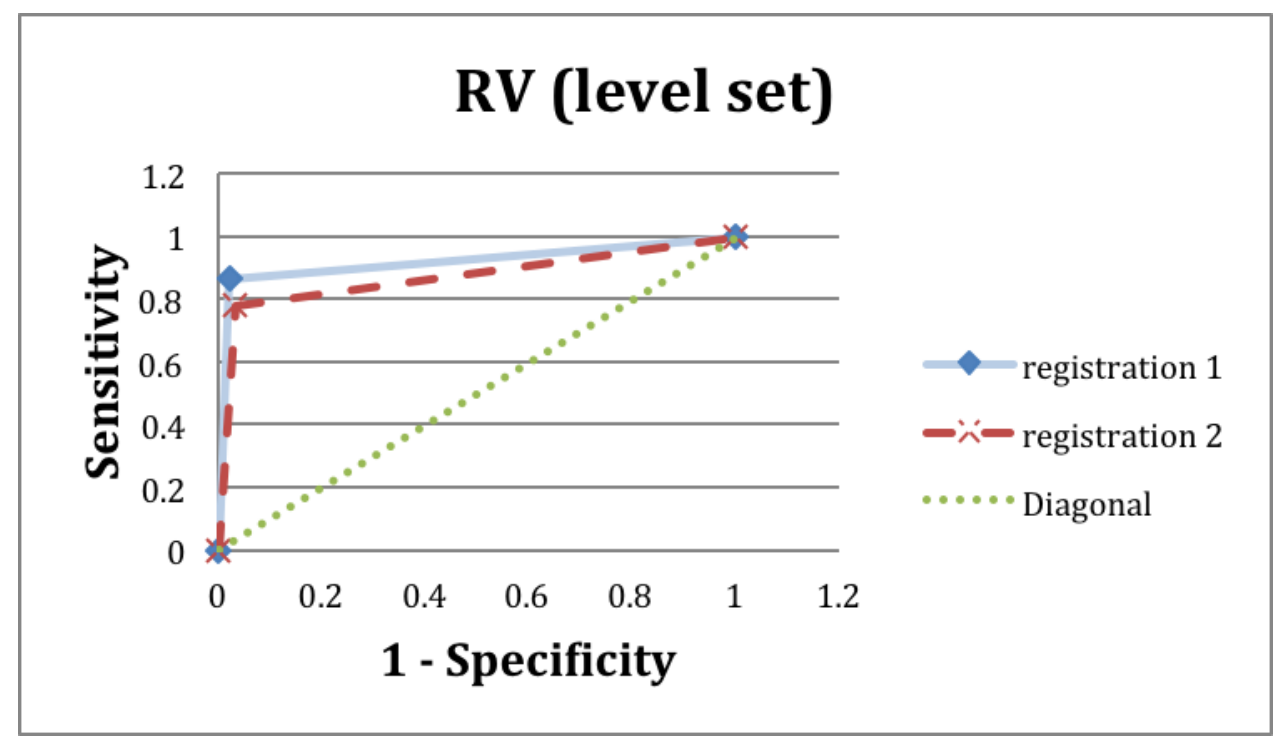

Fig 6: the ROC curve defines the difference between the two registration algorithms on RV segmentation using level set method

Table 3. Effect of initialization on LV segmentation using levelset method.

\begin{tabular}{|c|c|c|c|c|c|}
\hline & Slice level & $\begin{array}{c}\text { DM (mean } \\
\pm \text { SD) }\end{array}$ & $\begin{array}{c}\text { HD (mean } \\
\pm \text { SD) }\end{array}$ & $\begin{array}{c}\text { Sensitivity } \\
\text { (\%) }\end{array}$ & $\begin{array}{c}\text { Specificity } \\
(\%)\end{array}$ \\
\hline \multirow{2}{*}{$\begin{array}{c}\text { Initial contour } \\
\text { of current obtained } \\
\text { from pervious } \\
\text { frame }\end{array}$} & Base & $0.89 \pm 0.08$ & $5.77 \pm 3.24$ & 84.04 & 99.34 \\
\cline { 2 - 6 } & Mid & $0.73 \pm 0.08$ & $9.10 \pm 2.49$ & 66.20 & 99.34 \\
\hline $\begin{array}{c}\text { Initial contour } \\
\text { for each frame } \\
\text { obtained using } \\
\text { polar } \\
\text { transformation }\end{array}$ & Apex & $0.70 \pm 0.13$ & $5.69 \pm 2.25$ & 79.38 & 99.77 \\
\cline { 2 - 6 } & Base & $0.88 \pm 0.10$ & $6.79 \pm 4.10$ & 85.03 & 99.33 \\
\cline { 2 - 6 } & Mid & $0.81 \pm 0.09$ & $7.00 \pm 2.66$ & 73.76 & 99.58 \\
\hline
\end{tabular}


International Journal of Computers \& Technology

Table 4. Effect of initialization on RV segmentation using levelset method.

\begin{tabular}{|c|c|c|c|c|c|}
\hline & Slice level & $\begin{array}{c}\text { DM (mean } \\
\pm \text { SD) }\end{array}$ & $\begin{array}{c}\text { HD (mean } \\
\pm \text { SD) }\end{array}$ & $\begin{array}{c}\text { Sensitivity } \\
\text { (\%) }\end{array}$ & $\begin{array}{c}\text { Specificity } \\
(\%)\end{array}$ \\
\hline $\begin{array}{c}\text { Initial contour } \\
\text { of current obtained } \\
\text { from pervious } \\
\text { frame }\end{array}$ & Base & $0.86 \pm 0.06$ & $10.24 \pm 3.60$ & 91.86 & 96.79 \\
\cline { 2 - 6 } & Mid & $0.77 \pm 0.09$ & $10.03 \pm 2.86$ & 71.59 & 98.97 \\
\hline $\begin{array}{c}\text { Initial contour } \\
\text { for each frame } \\
\text { obtained using } \\
\text { polar } \\
\text { transformation }\end{array}$ & Base & $0.84 \pm 0.09$ & $12.50 \pm 6.48$ & 78.45 & 98.34 \\
\cline { 2 - 6 } & Mid & $0.80 \pm 0.10$ & $8.83 \pm 3.78$ & 81.42 & 98.64 \\
\hline
\end{tabular}

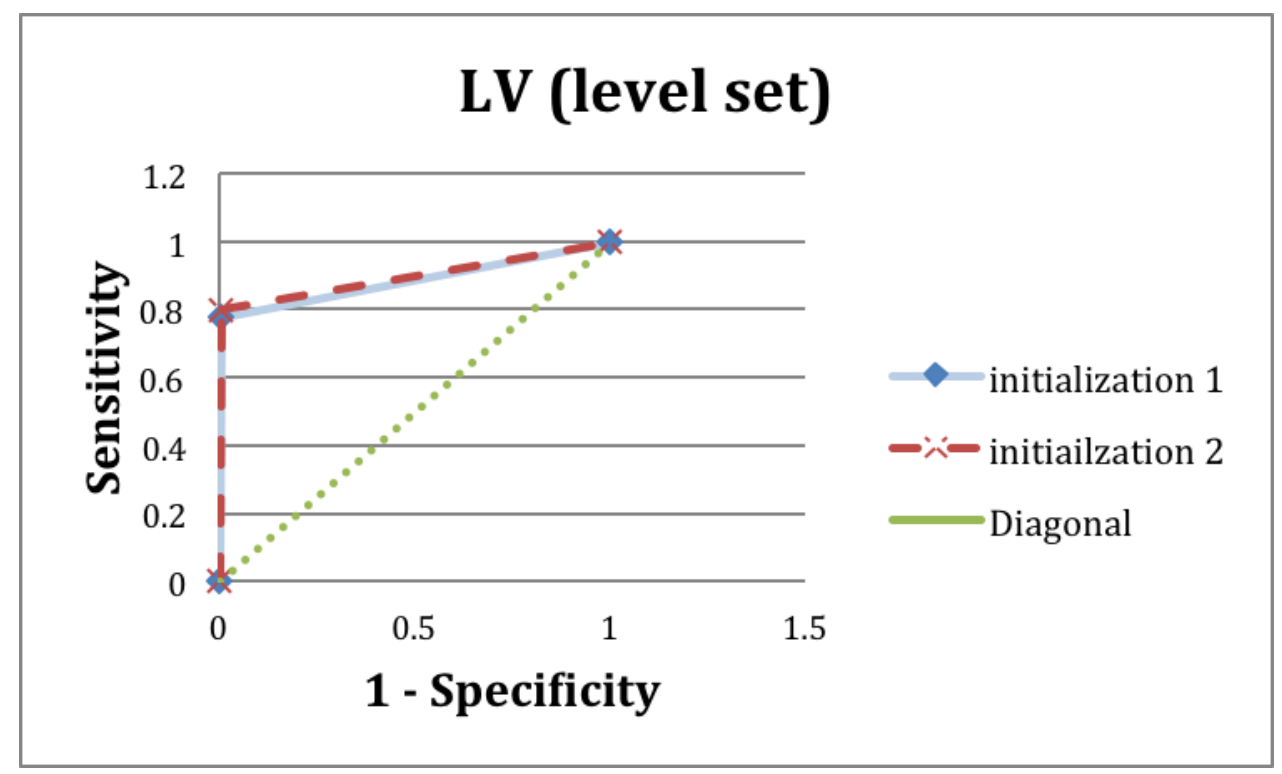

Fig 7: the ROC curve defines the difference between the two initialization algorithms on LV segmentation using level set method

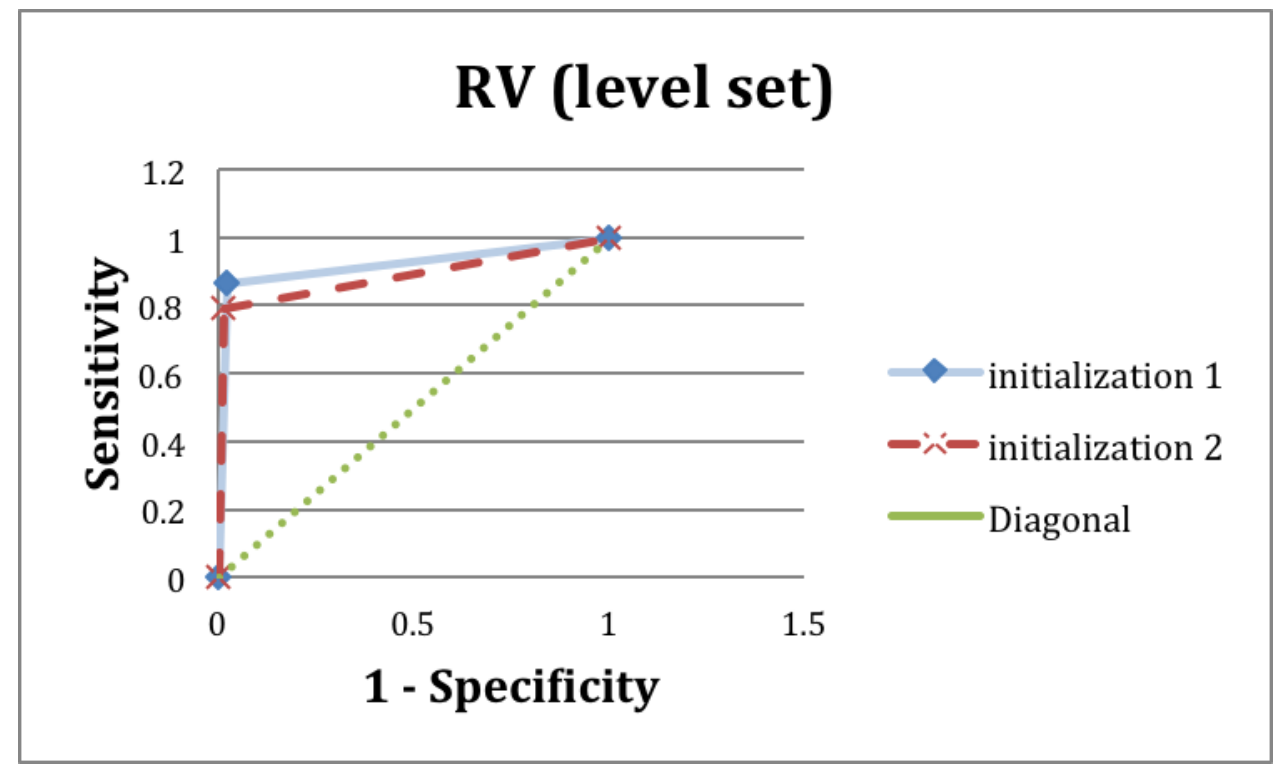

Fig 8: the ROC curve defines the difference between the two initialization algorithms on RV segmentation using level set method 


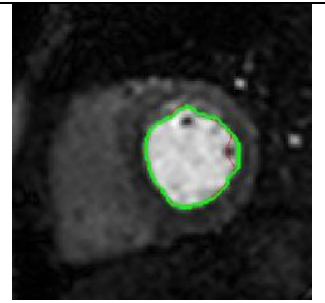

In ternation a
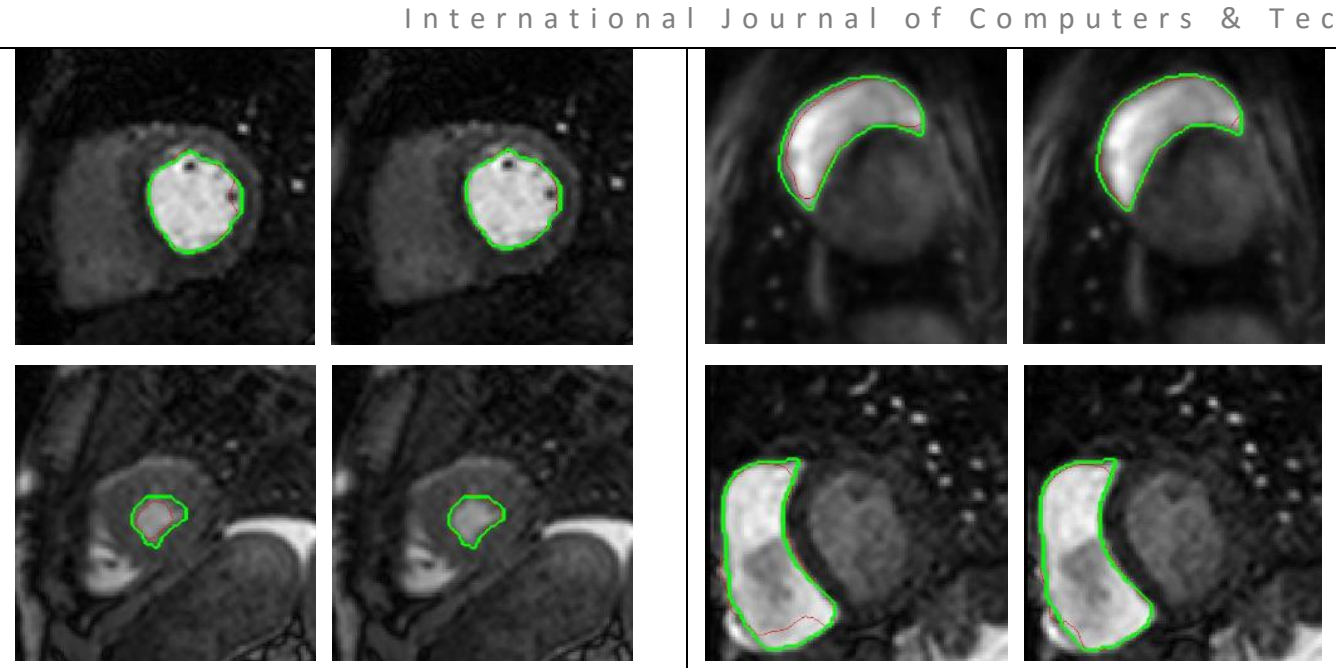

(a)

(b)

Fig 9: Segmentation results: first and second column related to first and second algorithm of initialization respectively using Level set method for (a) LV, and (b) RV. (red contour represent automatic segmentation and green contour represent the manual

\section{CONCLUSION}

In this study, we have presented a modified fully automatic workflow to segment the heart ventricles using levelset method and to highlight the effect of the registration and contour initialization of the cardiac ventricles on the accuracy of levelset segmentation. Short axis view of the cardiac perfusion MRI datasets was used. The proposed method consists of six main steps: denoising step, localization step, initial contour extraction, followed by registration, segmentation, and finally, the evaluation and validation of results. The segmentation accuracy has been improved, using registration in image domain algorithm, by $5 \%$, and $9 \%$ for LV and RV segmentation respectively. Furthermore, the system introduces a new step to extract a real shape of the LV for each slice instead of assuming it circle or ellipse. A new initialization for the levelset method based on polar representation of the images is presented that improves the accuracy of segmentation results from 0.77 to 0.82 , measured using Dice metric. In future, improving the accuracy of results by investigating more sophisticated methods for both the registration and segmentation steps is suggested. As our initial contour step allow us to train any algorithm automatically, and effectively, so we can use Active shape models (ASM) to improve the segmentation accuracy. In addition, generalizing the algorithm to work for any type of cardiac MRI Data.

\section{REFERENCES}

1. Mendis, S., Puska, P., and Norrving, B. 2011 Global atlas on cardiovascular disease prevention and control, World Health Org., 2-14.

2. McRobbie, D. , Moore, E., Graves, M., Prince, M. 2007 MRI: From Picture to Proton, Cambridge university press.

3. Grosgeorge, D., Petitjean, C., Caudron, J., Fares, J., Dacher, J. N. 2011 Automatic cardiac ventricle segmentation in MR images: A validation study, Int. J. Comput. Assist. Radiol. Surg., vol. 6, no. 5, 573-581.

4. Pluempitiwiriyawej, C., Sotthivirat, S. 2005 Active contours with automatic initialization for myocardial perfusion analysis, Conf. Proc. IEEE Eng. Med. Biol. Soc., vol. 3, pp. 3332-3335.

5. Shen, W., Kassim, A. a 2007 "A new scheme for automatic initialization of deformable models," IEEE Int. Conf. Image Process, vol. 4, pp. IV-289.

6. Wu, Y., Wang, Y., and Jia, Y. 2013 Segmentation of the left ventricle in cardiac cine MRI using a shape-constrained snake model, Comput. Vis. Image Underst., vol. 117, no. 9, pp. 990-1003.

7. Li, C., Xu, C., Gui, C., and Fox, M. D. 2010 Distance regularized level set evolution and its application to image segmentation, IEEE Trans. Image Process., vol. 19, no. 12, pp. 3243-3254.

8. Khalifa, F., Beache, G. M., Elnakib, a., Sliman, Gimel'farb, H. G., Welch, K. C., El-Baz, a. 2013 A new shape-based framework for the left ventricle wall segmentation from cardiac first-pass perfusion MRI, IEEE 10th Int. Symp. Biomed. Imaging, pp. 41-44.

9. Mousa, D., Zayed, N., and Yassine, I. 2016 The Impact of Polar based initialization and frame time curve selection on Left Ventricle short axis Perfusion MR Segmentation, ISMRM 24th Annual Meeting \& Exhibition, SMRT 25th Annual Meeting Conf..
$7225 \mid$ P a g e
council for Innovative Research
A ugust 2016
w w w. cirworld.com 
10. Sarode, M. V, and Deshmukh, D. P. R. 2010 Performance evaluation of rician noise reduction algorithm in magnetic resonance imaging, Journal of Emerging Trends in Computing and Information Sciences., vol. 2, pp. 39-44, .

11. Mousa, D., Zayed, N., and Yassine, I. 2014 Automatic cardiac MRI localization method, Cairo Inter. Biomed. Eng. Conf., pp. 153-157.

12. Yan, H., and Liu, J. G., 2008 Robust phase correlation based feature matching for image co-registration and DEM generation, Int. Arch. Photogramm. Remote Sens. Spat. Inf. Sci., no. B7, pp. 1751-1756.

13. Baswaraj, B. D., Govardhan, a, and Premchand, P. 2012 "Active contours and image segmentation: the current state of the art,” Glob. J. Comput. Sci. Technol. Graph. Vis., vol. 12, no. 11.

14. Ringenberg, J., Deo, M., Devabhaktuni, V., Berenfeld, O., Boyers, P., and Gold, J., Fast, accurate, and fully automatic segmentation of the right ventricle in short-axis cardiac MRI, Comput. Med. Imaging Graph., vol. 38, no. 3, pp. 190-201, Apr. 2014.

\section{Author' biography with Photo}

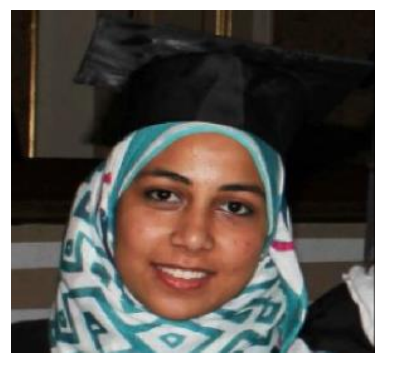

Doaa Mousa, has received her B.Sc. and M.Sc. degrees from the Biomedical Engineering Department at Cairo University, Egypt. She has been with the Computer Science and Systems Department at Electronics Research Institute, Cairo, Egypt since 2012 where she is currently a Researcher Associate. Her research interests include medical imaging and in particular Cardiac MRI and perfusion imaging, Neuroimaging, image pattern recognition, computer vision, and image processing.

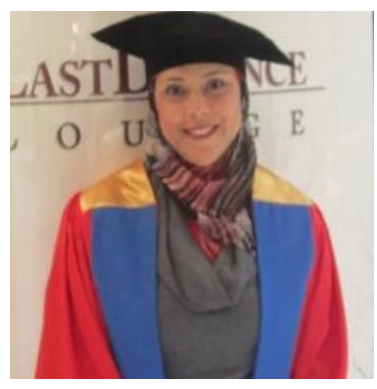

Dr. Nourhan Zayed, has received her Ph.D. in Electrical and Computer Engineering, University of Calgary, Calgary, Canada, where she also worked as a teaching assistant. She worked as an instructor in the Center for Adult and Continuing Education Department, American University in Cairo, Egypt in 2000- 2006. She has been with the Computer Science and Systems Department at Electronics Research Institute, Cairo, Egypt since 1999 where she is currently a Researcher. Along her career, she received several awards and recognitions including the record for research studentship funded by the multiple sclerosis program of the Hotchkiss Brain Institute, Calgary, Alberta, Canada (2008). She is a member of the IEEE, EMBS, ISMRM, and SPIE. Her research interests include medical imaging and in particular MRI and ultrasound, Neuroimaging, Multiple sclerosis and Optic neuritis imaging, multi-dimensional signal processing for biomedical applications, pattern recognition, computer vision, and image processing.

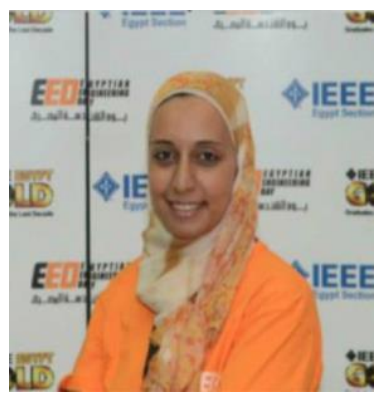

Dr. Inas Yassine, has received her PhD degree from West Virginia University USA. She is currently an Assistant Professor of Biomedical Engineering at Cairo University in addition to her positions as adjunct Assist. Prof. in School of Communication and Information Technology, Nile University and secretary of the IEEE- Egypt Section young professionals. Her research interest is in the area of Medical Image analysis, visualization, feature engineering, Machine Learning and big data analytics. 